\title{
Comparison of DEXA and QMR for Assessing Fat and Lean Body Mass in Adult Rats
}

\author{
Colette N. Miller ${ }^{\star}$, Tricia G. Kauffman ${ }^{\star}$, Paula T. Cooney, Keshia R. Ramseur, and Lynda M. \\ Brown ${ }^{\dagger}$ \\ Department of Nutrition, University of North Carolina at Greensboro, Greensboro, North Carolina, \\ USA
}

\section{Abstract}

There are several techniques used to measure body composition in experimental models including dual energy x-ray absorptiometry (DEXA) and quantitative magnetic resonance (QMR). DEXA/ QMR data have been compared in mice, but have not been compared previously in rats. The goal of this study was to compare DEXA and QMR data in rats. We used rats that varied by sex, diet, and age, in addition we compared dissected samples containing subcutaneous (pelt) or visceral fat (carcass). The data means were compared by focusing on the differences between DEXA/QMR data using a series of scatter plots without assuming that either method is more accurate as suggested by Bland and Altman. DEXA/QMR data did not agree sufficiently in carcass or pelt FM or in pelt LBM. The variation observed within these groups suggests that DEXA and QMR measurements are not comparable. Carcass LBM in young rats did yield comparable data once the data for middle-aged rats was removed. The variation in our data may be a result of different direct and indirect measures that DEXA and QMR technologies use to quantify FM and LBM. DEXA measures FM and estimates fat-free mass. In contrast, QMR uses separate equations of magnetic resonance to measure FM, LBM, total body water and free water. We found that QMR overestimated body mass in our middle-aged rats, and this increased the variation between methods. Our goal was to evaluate the precision of DEXA/QMR data in rats to determine if they agree sufficiently to allow direct comparison of data between methods. However DEXA and QMR did not yield the same estimates of FM or LBM for the majority of our samples.

\section{Keywords}

Dual Energy X-Ray Absorptiometry (DEXA); Quantified Magnetic Resonance (QMR); body composition; lean body mass; fat mass; Long-Evans rats

\section{Introduction}

Obesity is a rising concern, affecting approximately $30 \%$ of United States adults [1,2]. By some estimates, rates of overweight and obesity are expected to reach $86 \%$ by 2030 [3]. Body mass index (BMI) [4] and waist-to-hip ratio are two common ways to assess obesity

\footnotetext{
${ }^{\dagger}$ Corresponding Author: Lynda M. Brown, Department of Nutrition, University of North Carolina at Greensboro, P.O. Box 26170, Greensboro, NC 27402-6170, Telephone: 011336256 0320, Fax: 011336334 4129, 1mbrown6@uncg.edu.

These authors contributed equally to this work

Publisher's Disclaimer: This is a PDF file of an unedited manuscript that has been accepted for publication. As a service to our customers we are providing this early version of the manuscript. The manuscript will undergo copyediting, typesetting, and review of the resulting proof before it is published in its final citable form. Please note that during the production process errors may be discovered which could affect the content, and all legal disclaimers that apply to the journal pertain.
} 
[5]. Waist-to-hip ratio is based on the relative distribution of fat, categorizing body shape as gynoid or android. When fat is centralized in the abdominal region, risks for chronic diseases increase [6,7], including the risks for heart disease, stroke, type-2 diabetes, elevated blood pressure and plasma cholesterol, angina and respiratory problems [8].

While BMI and waist-to-hip ratio are useful for clinical assessment of obesity, more precise assessments of body composition, especially measures of lean body mass (LBM) and fat mass (FM), are important in clinical and research settings. There are several technologies used to measure body composition such as dual energy x-ray absorptiometry (DEXA) and magnetic resonance imaging (MRI), also called quantitative magnetic resonance (QMR). Body composition scanning using DEXA directly measures fat-free mass (FFM), but FM data is derived or indirectly measured. QMR uses differences in the nuclear magnetic resonance of hydrogen atoms and hydrogen density to directly measure FM, LBM, total body water and free water (fluids outside of tissue, e.g. urine and blood) [9]. In addition, LBM and FM are used to calculate other metabolic measures like energy expenditure, increasing the importance of accuracy and precision in body composition data [10].

Proximate analysis or carcass composition analysis (CCA) was used before DEXA and QMR became available. Studies comparing CCA to DEXA and QMR verified the newer methods in mice and rats (Table 1). However, DEXA/QMR has only been compared in mice, but the two scanning methods have not been compared in rats. Without a direct comparison of DEXA/QMR in rats it is not possible to know whether the methods give similar results, or if DEXA/QMR data sufficiently agree to allow comparisons between studies using DEXA or QMR. Because the methods have different strengths and the body mass of the animals affects the accuracy of the results [9], one purpose of the current study was to compare DEXA/QMR data in rats. While there are a few studies that report DEXA/ QMR in rats, these studies did not compare the methods, the authors used these methods to measure FM and distribution [11] or bone density [12-14].

QMR is affected by tissue hydration and animal size [9], so we included a heterogeneous group of rats to test some of the limits of DEXA/QMR comparisons for FM and LBM. The rats in this study were male and female Long-Evans rats that were young or middle-aged (retired breeders) and were fed a low-fat (LF) or high-fat (HF) diet. Our goals were to 1) evaluate the precision of DEXA/QMR data, 2) determine if DEXA/QMR data agree sufficiently to allow direct comparisons of data using either method. We hypothesized that data obtained from DEXA or QMR would measure LBM and FM precisely such that studies using them would reliably report body composition. We were less sure that the data would be in agreement given the differences in DEXA/QMR technologies.

\section{Materials and Methods}

\subsection{Animal care}

Rats from three ongoing experiments in the lab were used for this project. We used agematched male and female ( 3 month old) and retired breeder ( 1 year old at the time of sacrifice) Long-Evans rats obtained from Harlan Labs (Indianapolis, IN). Upon arrival they were given 1 week to acclimate to the facility before entering sex-specific colony rooms where they were singly housed in plastic tub cages with wood shaving cage fill. Rooms were temperature $\left(22 \pm 2{ }^{\circ} \mathrm{C}\right)$ and humidity controlled and kept on a 12:12 light/dark cycle (lights on at $0400 \mathrm{~h}$ ). Rats had free access to food and water and were fed a LF diet (17\% fat and $3.1 \mathrm{kcal} / \mathrm{g}$, Harlan Teklad \#7012; Indianapolis, IN), a phytoestrogen-free LF diet (11\% fat and $3.1 \mathrm{kcal} / \mathrm{g}$, Harlan Teklad \#7014; Indianapolis, IN) or a HF diet (40\% fat and $4.54 \mathrm{kcal} /$ g, Research Diets \#D03082706; New Brunswick, NJ) depending on the experimental design. The HF diet contains butter as the fat source and was selected to match the high intake of 
animal-derived fat in the US diet. Rats fed the HF diet were in experiments that lasted $24 \mathrm{~h}$, $72 \mathrm{~h}$, or $30 \mathrm{~d}$. We used a total of 132 rats, including 58 intact females, 43 males, and 31 ovariectomized (OVX) rats (14 OVX + estradiol (E2) and 17 OVX + vehicle (V)). Diet comparisons included 72 rats fed the HF diet further divided by length of time $24 \mathrm{~h}(\mathrm{n}=13)$, $72 \mathrm{~h}(\mathrm{n}=19)$, or $30 \mathrm{~d}(\mathrm{n}=40)$. The remaining 60 rats were fed one of the LF diets. Agematched males and females were middle-aged (retired breeders, $n=40$ ) or young ( 3 months old, $n=92$ ). Following euthanasia the rats were exsanguinated and dissected. Rats were dissected so that the skin and subcutaneous fat (pelt) are removed using the muscles of the trunk and appendages as a boundary for the remaining carcass as previously described [15]. The pelt contains the subcutaneous fat and the carcass contains the visceral fat. The pelt and carcass for each rat were stored in separate Ziploc bags and stored at $-80{ }^{\circ} \mathrm{C}$ until the experiments were completed. The University of North Carolina at Greensboro Institutional Animal Care and Use Committee approved all protocols for these experiments.

\subsection{DEXA calibration and measurement}

DEXA measurement was performed at the University of North Carolina Greensboro using a GE Lunar Prodigy Advanced System (GE Healthcare; Milwaukee, WI) and the data were analyzed by Encore 2007 Small Animal software (version 11.20.068). The system was calibrated according to manufacturer's instructions prior to the start of the experiment. Additionally, daily calibration was made and included a scan of pure coconut oil in a Ziploc bag that was $100 \%$ fat and an empty Ziploc bag as a negative control. This was an important test since al the samples were stored in Ziploc bags. Lastly, a previously measured carcass was scanned during calibration to determine if there was measurement variance on different days. The carcass scanned at the beginning of each day gave the same results indicating that the day of measurement did not affect the data.

All samples were stored at $-80{ }^{\circ} \mathrm{C}$ until the experiment was completed. Frozen samples were transferred in small batches to a lab refrigerator $\left(4^{\circ} \mathrm{C}\right)$ for a week and then allowed to come to room temperature on the day scheduled for DEXA measurement. The batches were determined by the amount of time reserved for DEXA analysis each week and all samples were run by Paula Cooney who is a trained DEXA technician. Each sample was laid flat on the surface of the DEXA table. The carcass was positioned so that the appendages were pointed in four corners and the tail laid in a u-shape ending near the right arm. The skull was placed in its approximate position. The pelt was laid in a single layer with the fur-side facing the table and the fat layer facing the beam. DEXA measurements were taken in duplicate and no significant variance between measurements was observed. Upon completion of the scan, the samples were returned to a $-80{ }^{\circ} \mathrm{C}$ freezer until all DEXA data were collected.

\subsection{QMR calibration and measurement}

Arrangements were made with the Woods/Seeley lab to provide the QMR analysis. Frozen samples were sent to Cincinnati in two shipments; the first contained the middle-aged rats and the young male and intact female rats, the second shipment contained the young OVX \pm E2 rats. Shipment procedures included taking samples from the -80 freezer and placing them directly into ThermoSafe shipping containers with 2" Styrofoam walls calibrated to hold temperature for $48 \mathrm{~h}$. The containers were packed with dry ice above and below the samples and shipped overnight to the University of Cincinnati Obesity Research Center.

QMR measurement was performed using an EMR014 model machine from Echo Medical Systems (EchoMRI technology; Houston, TX). Samples were analyzed using EchoMRI2004_1.1 software from the manufacturer. The specialized rat model can measure rats weighing as much as $1000 \mathrm{~g}$. Prior to each session the QMR was calibrated with calibration tubes provided by EchoMRI. Upon arrival the samples were brought to room 
temperature and duplicate measures were taken for each sample. The protocol at the Obesity Research Center involves taking a $3^{\text {rd }}$ measure if the first two differ by more than $10 \%$. None of the samples required a $3^{\text {rd }}$ measure.

\subsection{Data analysis}

Data analyses were performed using SPSS (version 17.0) and are presented as means with corresponding SEM so that the data can be compared directly for each methods by experimental groups. Using analysis to compare two methods [16,17], the difference between the DEXA-QMR data were plotted against the mean of the two methods (DEXA $+\mathrm{QMR} / 2$ ) in scatter plots. For the final scatter plot with little variation, standard deviation for the mean differences was obtained in addition to Pearson correlation coefficient. Significance was set at $\mathrm{p}<0.05$.

\section{Results}

\subsection{Comparison of DEXA and QMR in Long-Evans rats}

Using analysis to compare two methods [16,17], the difference between the DEXA-QMR data were plotted against the mean of the two methods (DEXA+QMR/2) in a scatter plot to determine whether the results center around zero. If the DEXA/QMR data were comparable, the differences would be small, center around the zero line, and be equally above and below it (Figure 1). DEXA/QMR data did not agree sufficiently in carcass and pelt FM (Figures 1A and B) or for pelt LBM (Figure 1D). The variation observed within these groups suggests that DEXA and QMR measurements are not comparable. Within the carcass LBM (Figure 1C), most points were centered around the zero line, however there was a small cluster of outliers. Looking at the raw data, the outliers contained all of the middle-aged rats. Removal of middle-aged rats from the data set reduced the variation seen in the scatter plot (Figure 2).

\subsection{Comparison of carcass LBM in young male and female rats}

Once the middle-aged rats were removed from the data set, the low variation between measurements observed in the carcass LBM suggests that DEXA/QMR data are comparable in young rats (Figure 2). This is supported further by the mean of the differences $(-0.63 \pm$ 1.41) and standard deviation of 13.29. Additionally, the Pearson's correlation coefficient of -0.05 between the pair differences and means was not significant $(\mathrm{p}=0.66)$.

\section{Discussion}

We report for the first time a comparison of QMR/DEXA methods for body composition in rats. Our study compared body composition data in subgroups of rats by sex, diet, and age, in addition to, comparisons for subcutaneous and visceral fat (Table 2). The scanning techniques were compared by focusing on the differences between DEXA/QMR data using a series of scatter plots (Figure 1) without assuming that either method is more accurate $[16,17]$. DEXA/QMR data did not agree sufficiently in carcass and pelt FM (Figures 1A and B) or for pelt LBM (Figure 1D). The variation observed within these groups suggests that DEXA and QMR measurements are not comparable.

Carcass LBM in young rats did yield comparable data once the data for aged rats was removed. One difference in the middle-aged rats was that the middle-aged males were the largest rats, with several weighing more than $700 \mathrm{~g}$, and this could be beyond the detection accuracy for DEXA and/or QMR. Body mass wasn't the only limitation in our comparison, middle-aged females weighing only $20-30 \%$ more than young females, had different values 
for FM and LBM as well. This indicates that studies using aged rats may not be comparable across methods.

DEXA and QMR quantify different aspects of the body. DEXA measures FM and gives an estimate that the rest is FFM. However, QMR uses separate equations of magnetic resonance to measure four entities FM, LBM, total body water and free water. We found that QMR overestimated body mass in our aged rats, i.e., when FM and LBM weights were added, total body mass was overestimated by $200 \mathrm{~g}$. This resulted in a large difference between mean pairs which added to the variation between DEXA/QMR (Table 2, Figure 1). Perhaps the direct measures of water explain why QMR data is affected by hydration as reported by Nixon and colleagues [9]. DEXA/QMR calculations were more variable in our middle-aged rats and it is possible that hydration differs in aged rats; however we did not measure hydration independently of the QMR data. The effects of age on hydration and how this may alter QMR data will require further investigation.

It is important to emphasize that DEXA and QMR did not yield the same estimates of FM or LBM for the majority of our samples (Table 2). Although this may be a result of the different conceptual estimates in DEXA/QMR, they both measure or estimate FM and FFM or LBM. What is puzzling is that these estimates should be measures for similar body depots, yet our data demonstrate clear differences. This differs from what was reported when DEXA/QMR were compared in mice. Tinsley and Taicher compared DEXA/QMR in mice and the correlation between the two methods was high, allowing them to conclude that QMR was a more precise method for mice $[18,19]$.

CCA was the gold standard for measuring body composition in rodent models before the QMR/DEXA technologies were developed. Thus each was compared initially to CCA to determine if they would be as accurate in research protocols. Studies that compared either scan to CCA in mice described over estimation of FM by DEXA [20] or QMR [21] (Table 1). Yet studies in rats comparing DEXA or QMR to CCA reported that QMR is more precise than CCA [9], and that DEXA is a more precise method than CCA for animals with a body mass greater than $200 \mathrm{~g}[22,23]$. It is difficult to interpret our data given these findings.

DEXA and QMR were validated in rats as methods that provide precise body composition data when compared to CCA; however our data comparing DEXA/QMR in rats demonstrates that these scanning techniques give results that differ. Additionally, DEXA/ QMR comparisons can be made in mice. Our data may represent a limitation in this comparison and perhaps indicate that comparisons are valid in the body mass range of mice (25-35 g), but this is lost in higher body mass ranges for rats (250-700 g). This hypothesis needs to be tested in future experiments.

It is possible that the pelt represents a low limit of detection for DEXA and/or QMR since this is skin with a layer of fat that is much smaller than the carcass. There should be very little LBM in the pelt, since all of the body muscles, bone and water would be in the carcass. As a result the variability in FM and LBM data may be a reflection of lower limits of detection. This is demonstrated in the variability of the scatter plots for pelt FM and LBM (Figures $1 \mathrm{~B}$ and D), DEXA consistently gave higher FM data and lower LBM than QMR. Additionally the $\mathrm{CV}$ data for pelts represents the data with the highest variability within methods providing the only percentages over 1.0 or 2.0 (Table 3 ).

DEXA and QMR data have not been previously compared in rats. This is an important experimental gap since measurements are affects by animal size. In the present study DEXA and QMR resulted in different estimates of FM and LBM in rats. FM data varied greatly between the two methods, while LBM was sufficiently similar to compare in young rats. 
This means that meta-analysis of body composition studies cannot compare data between methods.

It is possible that future studies will reveal a better way to report data from DEXA and QMR that provides data that more accurately represent FM and LBM. It is possible that future studies will reveal a better way to report data from DEXA and QMR that provides data that more accurately represent FM and LBM. Previous studies have concluded that there are optimal body mass ranges for DEXA and QMR, thus it is possible that our data provide clues to upper and lower limits for these optimal ranges. If the optimal range is identified for DEXA and QMR, this would provide researchers with a tool to select the best method for their experimental models.

\section{Acknowledgments}

We thank members of the Woods/Seeley lab at the University of Cincinnati Obesity Research Center, in particular Kathi Smith and Ken Parks, for providing the QMR data. Additionally, we thank Ken Gruber for statistical advice. This work was supported in part by NIH DK 17844 (Stephen C. Woods), USDA ARS grant NC06871(LMB) and a New Faculty Grant from the Office of Research and Economic Development at UNCG (LMB).

\section{References}

1. Steinbaum SR. The metabolic syndrome: an emerging health epidemic in women. Progress in Cardiovascular Disorders 2004;46:321-6.

2. Berthoud HR, Morrison C. The brain, appetite, and obesity. Annu Rev Psychol 2008;59:55-92. [PubMed: 18154499]

3. Wang Y, Beydoun MA, Liang L, Caballero B, Kumanyika SK. Will all Americans become overweight or obese? estimating the progression and cost of the US obesity epidemic. Obesity (Silver Spring, Md) 2008;16:2323-30.

4. Clinical guidelines on the identification, evaluation, and treatment of overweight and obesity in adults: executive summary. Expert Panel on the Identification, Evaluation, and Treatment of Overweight in Adults. Am J Clin Nutr 1998;68:899-917. [PubMed: 9771869]

5. Christian AH, Mochari H, Mosca LJ. Waist circumference, body mass index, and their association with cardiometabolic and global risk. J Cardiometab Syndr 2009;4:12-9. [PubMed: 19245511]

6. Despres JP. Cardiovascular disease under the influence of excess visceral fat. Crit Pathw Cardiol 2007;6:51-9. [PubMed: 17667865]

7. Wiklund P, Toss F, Jansson JH, Eliasson M, Hallmans G, Nordstrom A, et al. Abdominal and gynoid adipose distribution and incident myocardial infarction in women and men. Int J Obes (Lond).

8. Pi-Sunyer X. The medical risks of obesity. Postgrad Med 2009;121:21-33. [PubMed: 19940414]

9. Nixon JP, Zhang M, Wang C, Kuskowski MA, Novak CM, Levine JA, et al. Evaluation of a quantitative magnetic resonance imaging system for whole body composition analysis in rodents. Obesity (Silver Spring, Md) 2010;18:1652-9.

10. Kaiyala KJ, Morton GJ, Leroux BG, Ogimoto K, Wisse B, Schwartz MW. Identification of body fat mass as a major determinant of metabolic rate in mice. Diabetes 2010;59:1657-66. [PubMed: 20413511]

11. Weisinger HS, Begg DP, Egan GF, Jayasooriya AP, Lie F, Mathai ML, et al. Angiotensin converting enzyme inhibition from birth reduces body weight and body fat in Sprague-Dawley rats. Physiology \& behavior 2008;93:820-5. [PubMed: 18191966]

12. Badger AM, Blake S, Kapadia R, Sarkar S, Levin J, Swift BA, et al. Disease-modifying activity of SB 273005, an orally active, nonpeptide alphavbeta3 (vitronectin receptor) antagonist, in rat adjuvant-induced arthritis. Arthritis and rheumatism 2001;44:128-37. [PubMed: 11212150]

13. Badger AM, Griswold DE, Kapadia R, Blake S, Swift BA, Hoffman SJ, et al. Disease-modifying activity of SB 242235, a selective inhibitor of p38 mitogen-activated protein kinase, in rat adjuvant-induced arthritis. Arthritis and rheumatism 2000;43:175-83. [PubMed: 10643714] 
14. Hartman EH, Pikkemaat JA, Van Asten JJ, Vehof JW, Heerschap A, Oyen WJ, et al. Demineralized bone matrix-induced ectopic bone formation in rats: in vivo study with follow-up by magnetic resonance imaging, magnetic resonance angiography, and dual-energy X-ray absorptiometry. Tissue engineering 2004;10:747-54. [PubMed: 15265291]

15. Clegg DJ, Brown LM, Woods SC, Benoit SC. Gonadal hormones determine sensitivity to central leptin and insulin. Diabetes 2006;55:978-87. [PubMed: 16567519]

16. Bland JM, Altman DG. Statistical methods for assessing agreement between two methods of clinical measurement. Lancet 1986;1:307-10. [PubMed: 2868172]

17. Dallal G. Comparing Two Measurement Devices: Part I. 2000

18. Tinsley FC, Taicher GZ, Heiman ML. Evaluation of a quantitative magnetic resonance method for mouse whole body composition analysis. Obesity research 2004;12:150-60. [PubMed: 14742854]

19. Taicher GZ, Tinsley FC, Reiderman A, Heiman ML. Quantitative magnetic resonance (QMR) method for bone and whole-body-composition analysis. Anal Bioanal Chem 2003;377:990-1002. [PubMed: 13680051]

20. Nagy TR, Clair AL. Precision and accuracy of dual-energy X-ray absorptiometry for determining in vivo body composition of mice. Obesity research 2000;8:392-8. [PubMed: 10968731]

21. Jones AS, Johnson MS, Nagy TR. Validation of quantitative magnetic resonance for the determination of body composition of mice. Int J Body Compos Res 2009;7:67-72. [PubMed: 20467582]

22. Makan S, Bayley HS, Webber CE. Precision and accuracy of total body bone mass and body composition measurements in the rat using X-ray-based dual photon absorptiometry. Can J Physiol Pharmacol 1997;75:1257-61. [PubMed: 9431451]

23. Bertin E, Ruiz JC, Mourot J, Peiniau P, Portha B. Evaluation of dual-energy X-Ray absorptiometry for body-composition assessment in rats. The Journal of nutrition 1998;128:1550-4. [PubMed: 9732318] 


$$
\text { A }
$$
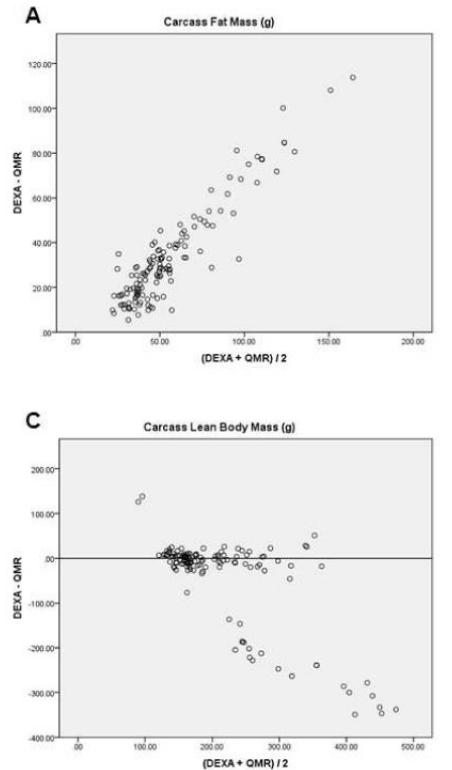

B
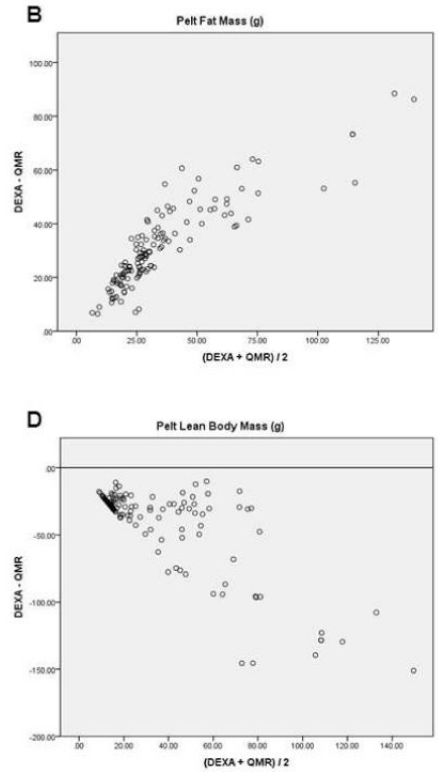

Figure 1. Variation of DEXA and QMR Data

Using analysis to compare two methods (Bland and Altman, 1986; Dallal, 2000), the difference between the DEXA-QMR data were plotted against the mean of the two methods (DEXA+QMR/2) to determine whether the results center around zero. If the DEXA/QMR data were comparable, the differences would be small, center around the zero line, and be equally above and below it. The results in figures $1 \mathrm{~A}, \mathrm{~B}$, and $\mathrm{D}$ are not centered at zero, indicating a large variation between DEXA/QMR data. Additionally, figures A and B only have points above the zero line indicating that the y-axis, DEXA- QMR is always positive. Therefore our DEXA data were consistently higher for carcass and pelt FM than the QMR data. Conversely, figure 1D only has negative values indicating that our QMR data were consistently higher for pelt LBM than the DEXA data. Most of the data in figure C, carcass LBM, centered near the zero line, but there were outliers with large variation. We examined our data set and discovered that the outliers were middle-aged rats. To check whether the data for young rats agreed sufficiently between the two methods, the data for the middleaged rats was removed and a new graph for carcass LBM in young rats was done (see Figure 2).

Rats were dissected so that the skin and subcutaneous fat (pelt) are removed using the muscles of the trunk and appendages as a boundary for the remaining carcass. This allows visceral and subcutaneous fat to be measured separately. The pelt contains the subcutaneous fat and the carcass contains the visceral fat.

Abbreviations: dual x-ray absorptiometry (DEXA); quantitative magnetic resonance (QMR); fat mass (FM); lean body mass (LBM). 


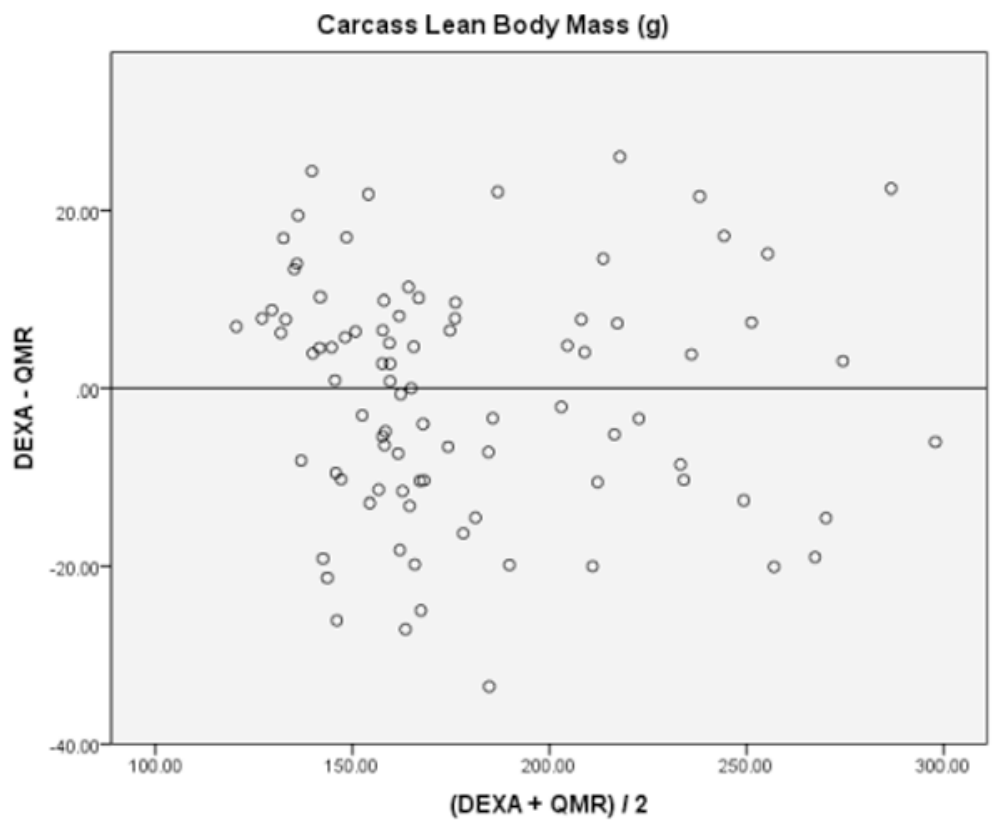

Figure 2. Variation of DEXA and QMR Data: Carcass LBM

Analysis to compare DEXA/QMR data in the carcass LBM of young rats was done after figure $1 \mathrm{C}$ revealed large variations in the data for middle-aged rats. This reduced the amount of variation observed and suggests that DEXA and QMR are comparable methods to measure carcass LBM in young rats. The carcass contains the body muscles, organs, bones and visceral fat without the skin and subcutaneous fat.

Rats were dissected so that the skin and subcutaneous fat (pelt) are removed using the muscles of the trunk and appendages as boundaries for the remaining carcass. This allows visceral and subcutaneous fat to be measured separately. The pelt contains the subcutaneous fat and the carcass contains the visceral fat.

Abbreviations: dual x-ray absorptiometry (DEXA); quantitative magnetic resonance (QMR). 


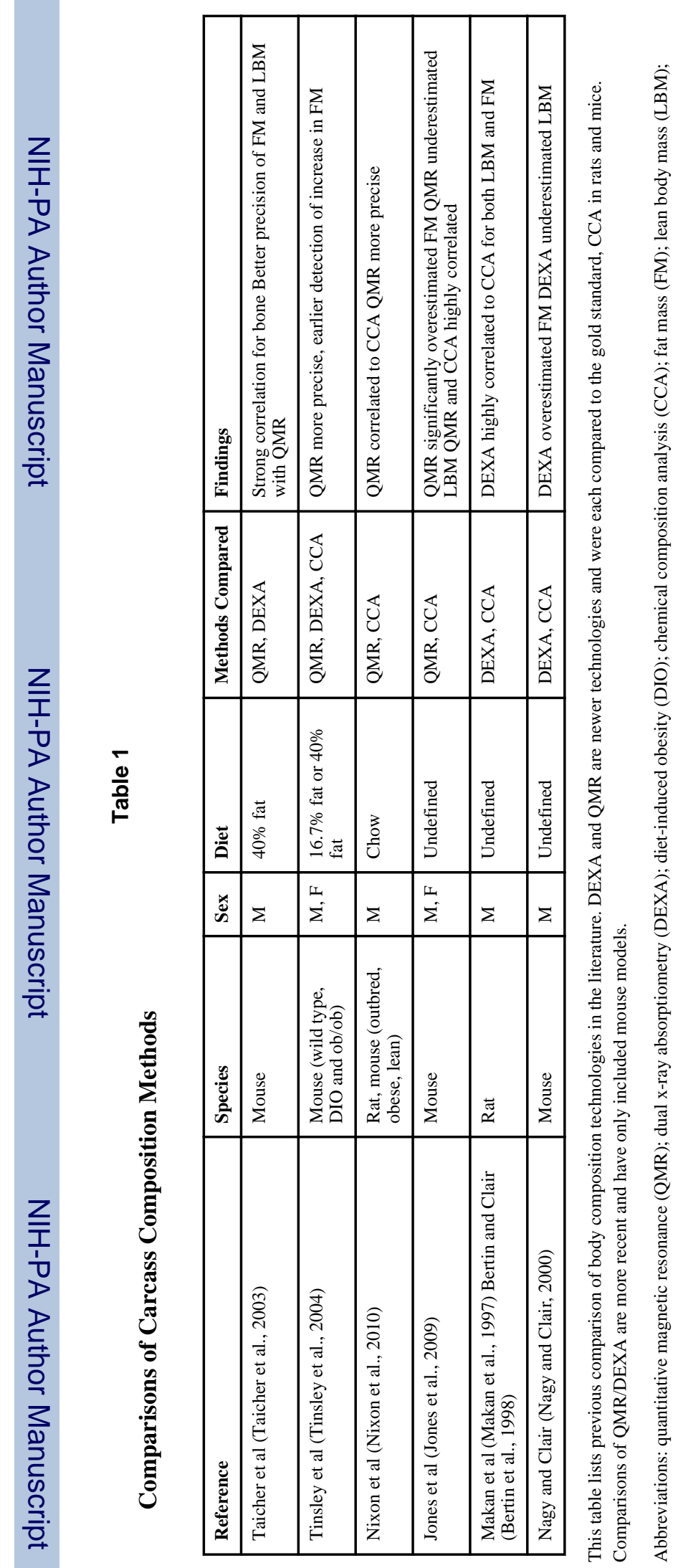

Physiol Behav. Author manuscript; available in PMC 2012 April 18. 


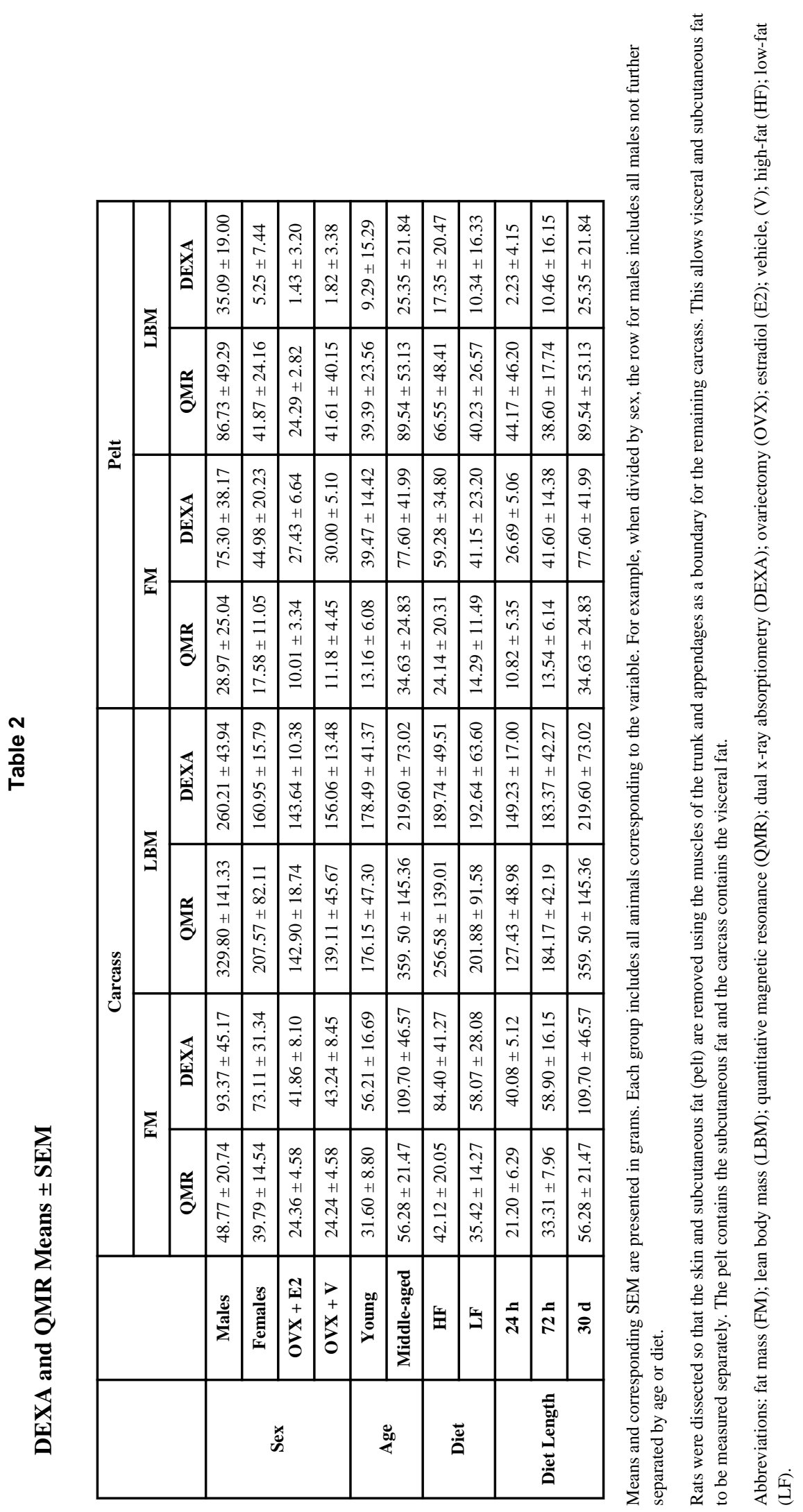

Physiol Behav. Author manuscript; available in PMC 2012 April 18. 


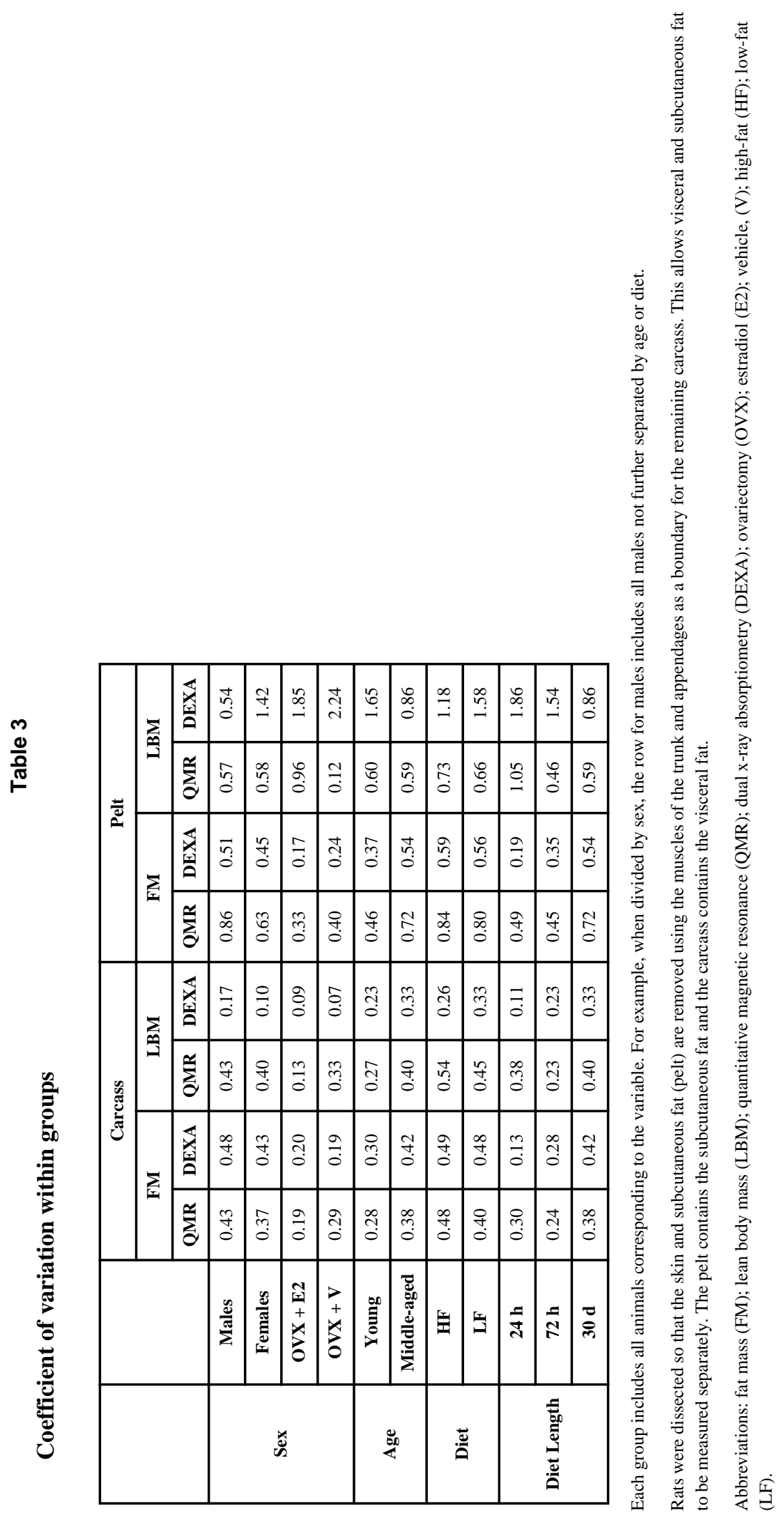

Physiol Behav. Author manuscript; available in PMC 2012 April 18. 